\title{
Multiple Event Source Routing (MESR) for Energy Efficient Wireless Sensor Network
}

\author{
Ayan Kumar Das \\ Calcutta Institute of Engineering and Management \\ 24/1A Chandi Ghosh Road, Kolkata-700040
}

\author{
Rituparna Chaki \\ West Bengal University of Technology \\ BF-142, Sector-I, Saltlake, Kolkata-700064
}

\begin{abstract}
The Wireless Sensor Network (WSN) is used to sense different events occurring at different sections of the remote network. The sensor nodes have limited battery power, and the nature of their deployment makes recharging almost impossible. To limit the power drain, efficient communication protocols are needed. This paper proposes Multiple Event Source Routing (MESR) for sending critical information by optimal path, so that it reaches the base station in shortest possible time. MESR sends regular or not-so-critical information in one of the multiple routing paths. MESR is capable of handling multiple events simultaneously. It defines an overhearing ratio in order to reduce an overhearing energy waste among selected paths. Thus MESR protocol improves reliability as well as network lifetime also.
\end{abstract}

\section{General Terms}

Routing in Wireless Sensor Network

\section{Keywords}

Overhearing ratio, Energy metric, Event path, Event priority, Congestion, Flooding

\section{INTRODUCTION}

Wireless Sensor Network is used to sense different types of events from different remote areas and communicate that event to all the nodes of the network as well as the base station also. Fast and reliable communication can help the base station to take corrective action against devastating events as soon as possible to save human civilization. Some algorithms are highly concentrated on finding out the optimal path to send the data packets consisting of event information. Although this results a quick packet transmission, the use of same path again and again will reduce the power of nodes of that path, as communication or data packet transmission between nodes is the main reason for power dissipation of the nodes. After some communication if the power of a node totally goes down, the node will be damaged and the path will be disconnected. New path creation will take extra time and also increase the overhead of the network. Thus energy efficient routing is a major challenge in WSN.

The protocol MESR is designed to make an energy efficient path to transmit information of events. Events may be of two types- devastating event and non devastating or regular event. The source node will send the information of devastating event through the optimal path, as the delivery of that information as early as possible to base station is more important than saving of power consumption. On the other hand when the regular event occurs the source node will create multiple paths keeping on mind that no two paths will be overlapped. The algorithm also maintains an overhearing ratio to restrict the overhearing effect between the paths, as it may cause the wastage of power. However among the multiple paths one path should be selected depending on the path selection probability. Thus no path will be selected again and again, which results in uniform power depletion through out the network. This scheme is capable of handling multiple events at a single instance. If a node receives different data packet of different event then it will forward that according to priority. Thus the algorithm helps for reliable transmission and at the same time energy efficient transmission also, which increases longevity of the overall network.

The remaining part of this paper is organized as follows: Section 2 deals with the review of state of the art routing topologies, section 3 gives a description of the proposed methodology, section 4 contains the simulation reports and section 5 is the concluding part.

\section{REVIEW WORK}

\subsection{Power Aware Routing Algorithms}

In this type of routing the main technique is focused on the effect of power efficient routing on the lifetime of multi hop wireless sensor networks (WSNs). All the nodes in the WSNs are divided into tiers. The nodes belonging to the highest tier are very important, because these nodes imply higher power consumption than that of nodes of any other tier. Obviously the batteries of nodes of 1 st tier depletes sooner than these of any other tier. As soon as the nodes of 1 st tier dies, the network becomes disconnected.

Energy Efficient Routing for Single Destination Flow redirection algorithm (FR) [1] is that type of algorithm in which we have a Largest longest length path (Lp) which is the path with largest capacity in terms of battery power and has less energy consumption per bit transmission than all other nodes in network and Smallest longest length path (Sp) which is the path with minimum capacity in terms of battery power and have higher energy consumption per bit transmission than all other nodes in network. It is the redirection based algorithm where some amount of flow is redirected from smallest longest length path ( $\mathrm{Sp}$ ) to largest longest length path (Lp). This algorithm firstly determines the two paths from source node to the destination which are to be involved in redirection. After that it calculates the amount of redirection $(€)$ and then $€$ amount of flow will be added to the largest longest length path Lp. After adding the algorithm checks one or possibly more loops in the path and then loops are removed link by link along the path so that the flows of the two paths can be properly incremented or decremented.

Maximum Residual Energy Path Routing (MREP) [2] algorithm is designed to augment the flow on the path whose minimum residual energy after the flow augmentation will be longest. This algorithm assumes that the topology of the 
network is static. Hence the results are applicable to networks which are either static like sensor network or whose topology changes slowly enough, but not applicable for ad hoc network whose topology changes very frequently. The algorithm used fixed information generation rates and required a priori knowledge of future information generation.

A Novel Power-Balancing Routing Scheme for WSN [3] is designed to detect the source of the network. In this algorithm the source node floods the event information along an event path and the other nodes send the queries at a regular time interval. A node sends the query to its neighbor consisting of maximum power and then the neighbor of neighbor and so on. When the query finds a node of event path then it detects the source node along with event path. The algorithm also guarantees that the query packet will not get an infinite loop by status checking mechanism and thus increasing the longevity of the overall network.

Split Multipath Routing (SMR) [4] is an on demand routing scheme that establishes and utilizes multiple routes of maximally disjoint paths. Providing multiple routes helps minimizing route recovery process and control message overload. This protocol uses a per-packet allocation scheme to distribute data packets into multiple paths of active sessions. This traffic distribution efficiently utilizes available network resources and prevents nodes of the route from being congested in heavily loaded traffic situations.

Energy Aware Source Routing (EASR) [5] protocol is designed to make an energy efficient paths from source to destination. Here multiple routing paths are selected. However one path will be selected for data transmission among those paths based on probability to be selected. In this algorithm the routing paths will be discovered with out overlapped. It also maintains an overhearing ratio to restrict the overhearing effect among multiple paths to reduce the energy waste.

Intelligent Energy Efficient Routing for Ad-Hoc Sensor Network by Designing QCS Protocol [6] states that depending on the type of information generated on a particular node or signal transmitted from a particular node to other node can be of three types- a) Regular information, b) Irregular Information, c) Devastating - Immediate Response Information. Here the network goes through mainly three stages - Nodes placing, Node activation and Node communication. In "QCS protocol" which stands for "QUERY-CHECKED-SOURCE" protocol, all the nodes of the network are of same type but all of them can behave in three different categories - QUERY NODE, CHECKED NODE and SOURCE NODE, depending on the situation. Signals are categorized into three types, 1.regular, 2.irregular \& 3.devastating depending upon the sensed value by the sensor. Network Initialization is done in the following way- A node is activated as "Q" node (Query node) randomly from all nodes in the network. The one non adjacent node of "Q" is chosen and denoted as "Q". This procedure continues until all the nodes nonadjacent to each other are chosen and denoted as "Q". Query node will query first and those nodes which will answer be the "C" nodes, i.e. checked nodes. At the next moment (after the time period "T") "C" nodes turn into "Q" and will repeat the procedure. The above procedure is true for type $1 \&$ type 2 signals. The type 3 signal will be flooded following Final Broad Cast-Petrol Flow protocol only, as it is an emergency \& make all adjacent as "S" and continue.

Load-Balanced Minimum Energy Routing (LBMER) [7] algorithm maximizes the network lifetime, as the energyconstraint sensor nodes in WSNs operate on limited batteries, it is a very important issue to use energy efficiently and reduce power consumption and for that it is essential to prolong each individual node's lifetime by minimizing the transmission energy consumption. This algorithm for WSNs considers both sensor node's energy consumption status and the sensor node's hierarchical congestion levels. It uses mixture of energy balance and traffic balance to solve the problem of congestion of WSNs and avoid the situation of congestion in sensor nodes using their energy at much higher rate and die much faster than the other nodes. The path for routing established by LBMER will not be very congested and the traffic will be distributed evenly in the WSNs. Simulation results verified that the LBMER performance is better than that of Min-Hop routing and the existing minimum energy routing scheme MTPR.

\subsection{Cluster Based Routing Algorithm}

In clustered network, nodes are clustered in the form of hierarchical structure. The advantage of cluster based method is improving routing efficiency, scalability, supporting QOS and saving power consumption in the nodes. Generally clustering transforms a physical network into a virtual network which has interconnected clusters.

Low Energy Adaptive Clustering Hierarchy (LEACH) [8] is also cluster-based protocol, which includes distributed cluster formation. LEACH randomly selects a few sensor nodes as cluster heads $(\mathrm{CHs})$ and rotates this role to evenly distribute the energy load among the sensors in the network. In LEACH, the cluster head $(\mathrm{CH})$ nodes compress data arriving from nodes that belong to the respective cluster, and send an aggregated packet to the base station in order to reduce the amount of information that must be transmitted to the base station. LEACH uses a TDMA/CDMA MAC to reduce intercluster and intra-cluster collisions. However, data collection is centralized and is performed periodically. Therefore, this protocol is most appropriate when there is a need for constant monitoring by the sensor network. A user may not need all the data immediately. Hence, periodic data transmissions are unnecessary which may drain the limited energy of the sensor nodes. After a given interval of time, a randomized rotation of the role of the $\mathrm{CH}$ is conducted so that uniform energy dissipation in the sensor network is obtained. The authors showed that only $5 \%$ of the nodes need to act as cluster heads.

Hybrid Energy-Efficient Distributed (HEED) routing [9] is also a clustering approach, which is one of the most recognized energy-efficient clustering protocols. It extends the basic scheme of LEACH by using residual energy and node degree or density. In HEED, the initial probability for each node to become a tentative cluster head depends on its residual energy, and final heads are selected according to the intra-cluster communication cost. The clustering process is divided into a number of iterations, and terminates with in a constant number of iterations. HEED achieves fairly uniform distribution of cluster heads across the network.

\subsection{Agent Based Routing}

Energy-Efficient Ant-Based Routing algorithm (EEABR) [10] is based on the Ant Colony Optimization (ACO). EEABR uses a colony of artificial ants that travel through the WSN looking for paths between the sensor nodes and a destination node, that are at the same time short in length and energyefficient, contributing in that way to maximize the lifetime of the WSN. Each ant chooses the next node to go with a probability that is a function of the energy of the nodes. When an ant reaches the destination node, it travels backwards trough the path constructed and updates the pheromone trail 
by an amount that is based on the energy quality and the number of nodes of the path. After some iteration the EEABR protocol is able to build a routing tree with optimized energy branches.

Ant-Colony Based Routing Algorithm (ARA) [11] used distance vector routing. Route discovery in ARA is done by broadcasting Forward Ants (FANT). ARA implements the packet sequencing mechanism to prevent packet loops. In ARA, destinations respond to multiple FANT packets received from the same node, thus supporting multi path routing.

\subsection{Query Driven Routing}

In Information-Driven Sensor Querying (IDSQ) [12], the querying node is capable of determining which node can provide the useful information with the advantage of balancing the energy cost. However, IDSQ does not specifically define how the query and the information are routed between sensors and the base station. Therefore, IDSQ can be seen as a complementary optimization procedure. Simulation shows that these approaches are more energyefficient than directed diffusion where queries are diffused in an isotropic fashion and reaching nearest neighbors first.

\subsection{Zone Based Routing}

The zone-based routing algorithm [13] generally follows a method in which max-min coordinate system is used. It is used for large scale networks and is fairly good to optimize the lifetime of the network. Zone-base routing is a hierarchical method where the area covered by the sensor network is divided into a small number of zones. Each zone has many nodes and thus a lot of redundancy occurs in routing a message through it. To send a message across the entire area it finds a global path from zone to zone and gives each zone control over how to route the message within itself. A local path for the message is computes within each zone so as to not decrease the power level of the zone too much.

\section{PROPOSED WORK}

\subsection{Basic Concept}

The objective of the proposed scheme is to transmit event information from source node to base station at minimum delay with optimized power consumption to increase the longevity of the overall network. The MESR algorithm first do the Event Categorization to set priority to events that has occurred in the vicinity of the nodes. For example if an earthquake occurs and its intensity is computed by the node in the range of 6.5 and above on the rector scale then treat that as a higher priority event, and if the intensity is in below the range of 6.5 then treat that as a lower priority event. The data packets with higher priority event will be sent through the shortest path from source node to base station so that it can take very less time and we are not concerning about the power at that time. On the other hand in case of lower priority event it will construct at least two paths that are not overlapped. According to EASR [9] protocol each path should keep proper distance from each other to avoid the overhearing effect among paths. In SMR the intermediate nodes forward the duplicate packets that traversed through a different incoming link, instead of dropping every duplicate RREQs and the new arriving RREQ has to also satisfy the condition that the hop count of the duplicated packets is not larger than that of the received RREQ. The proposed algorithm uses the same technique of route generation as of SMR [18].
The algorithm assumes that all of the nodes collect neighbor node IDs for the purpose of Route Reply. In the on demand routing protocol, the node IDs on the entire path are recorded in the RREP and hence the intermediate nodes can forward the RREP using this information. MESR requires two additional information for establishing energy efficient multiple paths - (a) The residual energy of all intermediate nodes is recorded in the RREP and (b) The neighbor list of each node is recorded in the RREP.

Now for Route Selection source node will store the first received RREP in the routing cache. After that when it will get other RREP, the source will decide whether the path mentioned by second RREP will be selected or not. The conditions that will drop a RREP are - (a) The path of new arriving RREP is overlapped with already selected paths and (b) The neighbor list of new arriving RREP is overlapped with already selected paths. The first condition is absolutely necessary when the second one is flexible. We can adjust a threshold of Overhearing Ratio defined as-

$$
\mathrm{R}_{\mathrm{k}}=\frac{\sum_{\mathrm{x} \in \mathrm{S}_{\mathrm{new}}\left|\mathrm{N}_{\mathrm{x}} \cap \mathrm{S}_{\mathrm{k}}\right|}\left|\mathrm{S}_{\mathrm{k}}\right|}{}
$$

Where, $R_{k}$ is the overhearing ratio of kth path which is already used for data transmission by source node. $\mathrm{N}_{\mathrm{x}}$ is the neighbor list of the node $\mathrm{x} . \mathrm{S}_{\mathrm{k}}$ is the set of intermediate nodes which are included in the kth path. $S_{\text {new }}$ is the set of intermediate nodes of new arriving RREP and $\left|S_{k}\right|$ is the number of elements of set $S_{k}$. One path among the multiple selected paths will be chosen based on the following probability, ensuring that none of the paths is used all the time to prevent energy depletion.

$$
\mathrm{P}_{\mathrm{n}}=\mathrm{E}_{\mathrm{n}} / \sum_{\mathrm{k}=1}^{\mathrm{S}} \mathrm{E}_{\mathrm{k}}
$$

Where, $P_{n}$ is the probability of nth path, $E_{n}$ is the energy metric of the nth path and $\mathrm{S}$ is the number of whole selected routes. The energy metric $\mathrm{E}_{\mathrm{n}}$ for $\mathrm{nth}$ path can be defined as-

$$
E_{n}=\left(R_{n}-L_{n}\right) / d_{n}
$$

Where, $R_{n}$ is the total residual energy of all nodes on the $n$th path. $L_{n}$ is the load of nth path, i.e., the energy required to transmit all the traversing data packets on that nth path, and $d_{n}$ is the distance or length of nth path.

At a time multiple events may occur in different nodes. Then it will have different source nodes. Every source node will start sending data packets of different information of different priority. Every source node will send the information by shortest path if the event priority is high. Though for low priority events every source node will select multiple paths considering the overhearing ratio for that path and then select one according to path probability, one source node never concerns about the path of other source nodes.

Now it is clear that the algorithm is trying to send devastating information very fast so that the base station can take necessary action and send non devastating information by checking the power of neighbor nodes so that the longevity of the overall network may increase. 


\subsection{Data Dictionary}

Table 1. Variables list

\begin{tabular}{|c|l|}
\hline $\begin{array}{c}\text { Variable } \\
\text { Name }\end{array}$ & \multicolumn{1}{|c|}{ Description } \\
\hline $\mathrm{N}$ & Total Number of nodes \\
\hline $\mathrm{P}$ & Initial power for all the nodes \\
\hline $\mathrm{Ep}$ & Event priority value \\
\hline $\mathrm{R}_{\mathrm{k}}$ & Overhearing Ratio \\
\hline $\mathrm{P}_{\mathrm{n}}$ & Probability to select a path \\
\hline $\mathrm{E}_{\mathrm{n}}$ & Energy Metric \\
\hline $\operatorname{arr}[]$ & $\begin{array}{l}\text { An array consists of the } \\
\text { connections between the nodes }\end{array}$ \\
\hline s_path[] & $\begin{array}{l}\text { List of all the nodes for shortest } \\
\text { path from a source node }\end{array}$ \\
\hline $\begin{array}{c}\text { power[node_i } \\
\mathrm{d}][\text { [energy] }\end{array}$ & $\begin{array}{l}\text { An array consists of initial power } \\
\text { of each node }\end{array}$ \\
\hline status[node_i & $\begin{array}{l}\text { Visited nodes are stored in this } \\
\text { array }\end{array}$ \\
\hline $\mathrm{d}]$ & $\begin{array}{l}\text { List of multiple paths from source } \\
\text { to base station }\end{array}$ \\
\hline Path[ ] & The distance or length of nth path. \\
\hline $\mathrm{d}_{\mathrm{n}}$ & $\begin{array}{l}\text { Total distance traveled during one } \\
\text { simulation }\end{array}$ \\
\hline Total
\end{tabular}

\subsection{Description}

\subsubsection{MESR ( )}

Step 1: Begin.

Step 2: Read $\mathrm{n}$ ( $\mathrm{n}$ is total number of nodes) and $\mathrm{p}$ ( $\mathrm{p}$ is initial power for all the nodes)

Step 3: Create the network with $\mathrm{n}$ nodes and set the initial value $\mathrm{p}$ for every node.

Step 4: The source node $m$ sense the event and set the priority value according to event intensity as ep : $2 / 1$ (high/low)

Step 5: If ep=2 then do-

a) Find the shortest path from source node $m$ to base station and store all the nodes of this path in the array s_path[ ].

b) $\quad I^{*}$ Reduce the power to transmit the packet from source node $\mathrm{m}$ to base station and find out the remaining average power of the network. */ Call function Reduce_Power ( )

Step 6: Else

a) Send the RREQ packet to the neighbors of source node (m).

b) After getting the RREP packet store the path information in the routing cache of source node.

c) Repeat following steps for $\mathrm{n}$ times ( $\mathrm{n}$ is the number of RREP packets of source $m$ )

i) Set the Overhearing ratio $R_{k}$ according to equation (i)

ii) $\quad$ If $R_{k}<0.4$ Then

Select the path and store it in the array path[ ].

End loop

d) Find the Energy metric $E_{n}$ for each path of the array path[ ].

e) Find Probability $P_{n}$ according to equation (ii) for choosing a path from array path[].

f) Send the data packet via selected path.

g) Call function Reduce_Power ( )

Step 7: End.

\subsubsection{Reduce_Power ( )}

//Reduce the power to transmit the packet from source node $\mathrm{m}$ to base station

Step 1: Begin

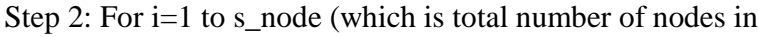
s_path[ ]) repeat step (a)

a) For $\mathrm{j}=1$ to $\mathrm{n}$ repeat the following step If s_path[i] = power[1][j], then

Set power[2][j]=power[2][j]-trans_power

// Find the average power of the network

Step 5: For $\mathrm{i}=1$ to $\mathrm{n}$ repeat the following Set total $=$ total + power[2][i]

Step 6: Set avg_power=total $/ \mathrm{n}$

Step 7: Return

\section{PERFORMANCE ANALYSIS}

To analyze the performance of the algorithm a network with nine sensor nodes has created. The initial power for every node is considered to 50 units. To simulate the algorithm 5 sources and 5 destinations are selected randomly. Each source starts data transmission in random time and it will stop after 100 seconds. The simulation parameters are given in the following table.

Table 2. Parameter list

\begin{tabular}{|l|l|}
\hline \multicolumn{1}{|c|}{ Parameters } & \multicolumn{1}{c|}{ Description } \\
\hline Network size & 15 nodes \\
\hline Initial energy & 50J per node \\
\hline MAC Protocol & IEEE 802.15.4 \\
\hline $\begin{array}{l}\text { Power } \\
\text { consumption }\end{array}$ & $\begin{array}{l}\text { Equivalent to packet size } \\
\text { and distance }\end{array}$ \\
\hline $\begin{array}{l}\text { Number of high } \\
\text { priority message }\end{array}$ & At least 10 \\
\hline
\end{tabular}

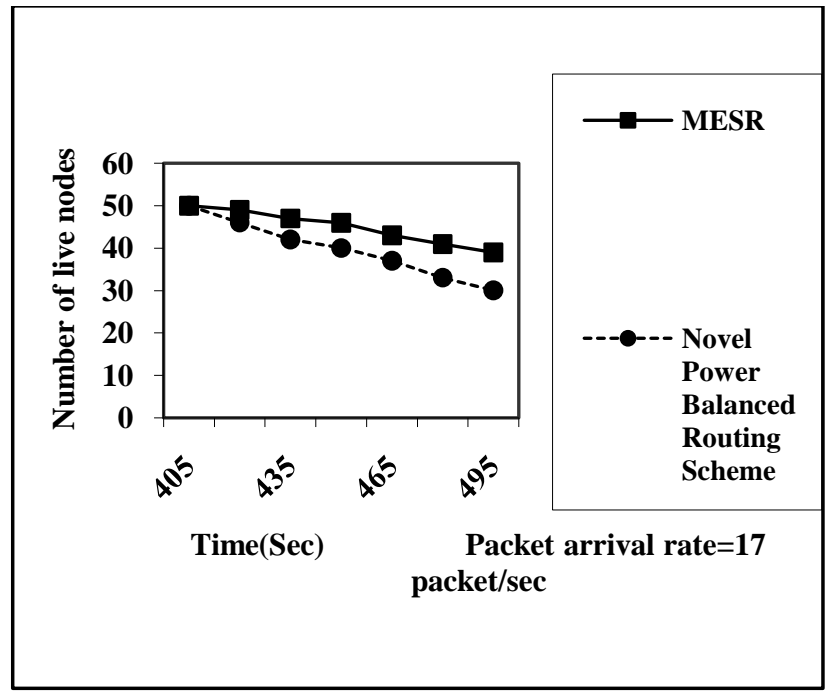

Figure 1: Number of live nodes in networks

Figure 1 shows that the number of live nodes after certain time interval from the start of the simulation for both MESR and the earlier scheme- 'A novel Power Balanced Routing Scheme for WSN' [2]. In that earlier protocol, the source node creates an event path and the other nodes sends the query packets to know the event. When the query packet is moving it will find the neighbor node which has the maximum power. Whatever, the above figure is showing that after sending some 
packets the number of live nodes for MESR algorithm is greater than that of the earlier proposed scheme. Thus MESR is increasing the longevity of the overall network. In this simulation, the threshold value of overhearing ratio is 0.4 , i.e. the overhearing effect is allowed only $40 \%$ of the entire path.

If we apply different threshold value of overhearing ratio then the performance of MESR would be changed. The following figure shows the number of live nodes of MESR according to the threshold of overhearing ratio.

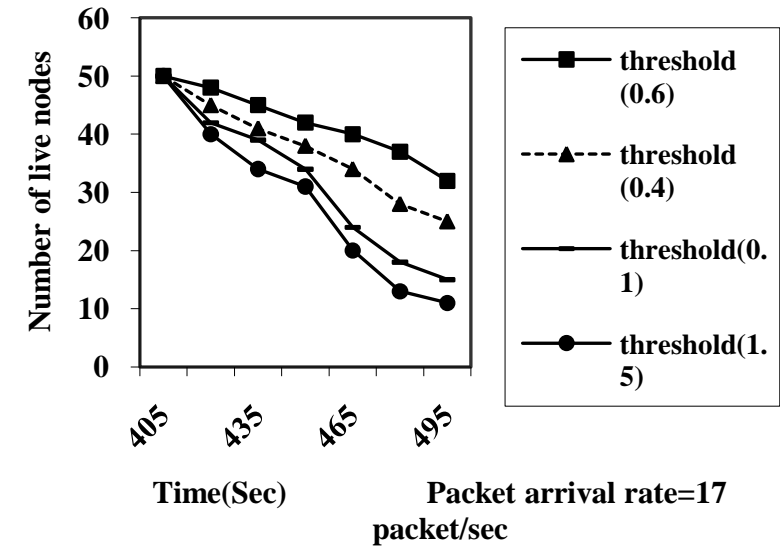

Figure 2: Number of live nodes according to threshold of overhearing ratio of MESR

The large threshold value means that most of the paths can be selected, which may cause a high overhearing effect and also wastage of energy. Otherwise the small threshold value means that the overhearing effect is not allowed strictly. Though the small threshold value gives the better performance, if the threshold is extremely small, it will always select only one path. It may cause more power depletion of the nodes of that path only and also increase the congestion of the network. Thus adjusting threshold level is very important for this protocol. Figure 2 shows that the threshold value 0.4 can be accepted, as it is giving better performance for all cases.

\section{CONCLUSION}

The event routing in optimal path has been an active area for researchers. The review of state of the art research has led to the observation that routing of not-so-critical information along the shortest possible path often leads to power drainage. This paper proposes MESR: Multiple Event Source Routing, which uses alternative routes for not-so-critical events towards the sink node. This helps in power saving of shortest possible routes ensuring speedy delivery of critical event information to the sink node. The authors are now busy with checking the performance of MESR against varying parameters.

\section{REFERENCES}

[1] Sourabh Jain, Praveen Kaushik and Jyoti Singhai, "Greedy Heuristic Based Energy Efficient Routing in Wireless Sensor Network", in proceedings of Communications in Computer and Information Science, 2011, Volume 203, Part 1, 282-292, DOI: 10.1007/9783-642-24037-9_27.
[2] J.QiangFeng and D.Manivannan, "Routing protocols for sensor networks", in Consumer Communications and Networking Conference, CCNC 2004.First IEEE.2004.

[3] Ayan Kumar Das, Dr. Rituparna Chaki, "A Novel Power Balancing Routing Scheme for WSN" in Proceedings of The Third International Conference on Wireless \& Mobile Networks (WiMo-2011), Ankara, Turkey will be published by Springer (LNCS) in Communications in Computer and Information Science (CCIS) Series,IssueJune, 2011.

[4] S.J. Lee and M. Gerla, "Split Multipath Routing with Maximally Disjoint Paths in Adhoc Network", ICC 2001.

[5] Do-Youn Hwang, Eui-Hyeok Kwon and Jae-Sung Lim, "An Energy Aware Source Routing with Disjoint Multipath Selection for Energy Efficient Multihop Wireless Adhoc Networks", F Boavida et al (Eds.): NETWORKING 2006, LNCS 3976, pp. 41-50, International Federation for Information Processing, 2006.

[6] Ayan Kumar Das, Debaditya Ghosh, Pritam Majumder, "Intelligent Energy Efficient Routing For Ad Hoc Sensor Network by Designing QCS Protocol" in Proceedings of The Second International Workshop on Adhoc, Sensor and Ubiquitous Computing (ASUC-2011) will be published by Springer (LNCS) in Communications in Computer and Information Science (CCIS) Series,IssueJune,2011.

[7] GONG Ben-can LI La-yuan JIANG Ting-yao XU Shouzhi, "Distributed Spanning Tree-Based Routing Protocol for Wireless Sensor Networks", MICROELECTRONICS \& COMPUTER, 2008, 25(11).

[8] W.R.Heinzelman, A.Chandrakasan, and H.Balakrishnan, "Energy Efficient Communication Protocol for Wireless Microsensor Networks", In Proceedings of the $33^{\text {rd }}$ Hawaii International Conference on System Sciences, 2000.

[9] Younis,O.; Fahmy, S.; Dept. of Comput. Sci., Purdue Univ., West Lafayette, IN, USA, "HEED: a hybrid, energy-efficient, distributed clustering approach for ad hoc sensor networks", appears in Mobile Computing IEEE Transactions on Volume: 3 Issue: 4, ISSN: 15361233 Issue Date: Oct.-Dec. 2004.

[10] Tiago Camilo, Carlos Carreto, Jorge Sá Silva and Fernando Boavida, "An Energy-Efficient Ant-Based Routing Algorithm for Wireless Sensor Networks", Lecture Notes in Computer Science, Volume 4150/2006, 49-59, DOI: 10.1007/11839088_5, 2006.

[11] M. Gunes, U. Sorges, I. Bouazizi, "ARA- the ant colony based routing algorithm for MANET",ICPP Proc of the 2002.

[12] S. Slijepcevic and M. Potkonjak, "Power efficient organization of wireless sensor networks", in IEEE International Conference on Communications", Helsinki, Finland, June 2001.

[13] Karim,L., Nasser,N.; ElSalti,T.; Sch. of Comput. Sci., Univ. of Guelph, Guelph, ON, Canada, "Efficient Zonebased Routing Protocol of Sensor Network in agriculture monitoring systems", Communications and Information Technology (ICCIT), ISBN: 978-1-4577-0401-7, Issue Date: 29-31 March 2011. 